\title{
Retos y oportunidades del diseño participativo en tecnología educativa
}

\section{Challenges and opportunities of participatory design in educational technology.}

(iD) Begoña Gros; bgros@ub.edu
Universidad de Barcelona (España)

D Eva Durall; eva.durall@aalto.fi

Aalto University (Finland)

\section{Resumen}

El diseño participativo está teniendo una importante incidencia en el diseño de entornos de aprendizaje mediados por tecnología. Cada vez es más frecuente utilizar los enfoques participativos y de codiseño para involucrar a docentes y a estudiantes en el diseño de la tecnología para generar soluciones centradas en las necesidades de las personas que se verán afectadas al utilizarlas. El diseño participativo pretende involucrar a los grupos afectados desde el inicio del diseño, lo que implica tener en consideración la complejidad que supone dar voz a personas con conocimientos, necesidades y roles diferentes. El objetivo principal de esta contribución es analizar el papel actual del diseño participativo con relación a la investigación e innovación en tecnología educativa. Se analiza el origen de este tipo de enfoque y las aplicaciones más habituales en el ámbito educativo. Posteriormente, se describen los métodos que facilitaran la comunicación y colaboración entre las diseñadoras y participantes, se analizan los principios clave en el diseño participativo, y, finalmente, se exponen las implicaciones del diseño participativo para la investigación e innovación en tecnología educativa.

Palabras clave: diseño participativo, codiseño, diseño de aprendizaje, tecnología educativa

\begin{abstract}
In the last decade, participatory design is having a major impact on the design of technology for learning. It is increasingly common to use participatory and co-design approaches to involve teachers and students in the design of technology to generate solutions focused on their needs. Participatory design aims to involve the final users during the whole process, which requires to develop strategies to work with and give voice to people with different knowledge, needs and roles. The aim of this article is to analyse the use of participatory design in educational technology. Firstly, we described the origin of this type of approach and the most common applications in the educational field. Secondly, we analyse the methods that facilitate communication and collaboration between designers and participants, as well as the key principles of participatory design. Finally, we discuss the implications of participatory design for research and innovation in educational technology.
\end{abstract}

Keywords: participatory design, co-design, learning design, educational technology 


\section{ORÍGENES Y TENDENCIAS PRINCIPALES EN EL DISEÑO PARTICIPATIVO}

En el campo del diseño participativo, podemos referirnos a dos tendencias principales: la tradición escandinava y la norteamericana. La primera tiene su origen en la década de los 70 en los países del norte de Europa, como parte de lo que se conoció posteriormente como el movimiento de la democracia laboral. En este contexto, sindicatos de empresas, desarrolladores de sistemas informáticos e investigadores iniciaron una serie de acciones colectivas con la intención de poner en cuestión el tipo de cambios producidos por la automatización de múltiples procesos en el lugar de trabajo. Como señala Ehn (1988), se trataba de crear nuevas visiones de los espacios y procedimientos de trabajo, apoyados por tecnología incorporando la perspectiva de los trabajadores; usuarios de estas nuevas tecnologías en su propio contexto. Esta estrategia permitía además capacitar a los trabajadores y potenciar sus habilidades de forma creativa (Robertson y Simonsen, 2012). El diseño participativo consistió en diseñar tecnologías de la información que permitirían a las personas cambiar y desarrollar aún más sus prácticas de trabajo para incorporar tanto el uso de sistemas informáticos como las mejores condiciones de trabajo. Sus raíces en el movimiento de la democracia en el lugar de trabajo significaron que aquellos cuyas nuevas prácticas de trabajo estaban siendo diseñadas se comprometieron centralmente en el proceso. Este compromiso fue definido y habilitado con el desarrollo de nuevos métodos de diseño, herramientas y técnicas destinadas a posibilitar que todos los involucrados en el proceso de diseño pudieran imaginar y avanzar hacia nuevas visiones del futuro.

Desde el principio, el diseño participativo ha tenido una orientación política, porque se considera no sólo una forma de mejorar la experiencia de los trabajadores, sino, sobre todo, como un movimiento hacia la democratización en el trabajo. En un artículo más reciente, Ehn (2008) describe la evolución del diseño participativo en los países nórdicos y destaca que ha habido un cambio muy importante ya que el diseño participativo ha pasado de estar centrado en el ámbito laboral a orientarse hacia el ámbito comunitario con el objetivo de incidir en los procesos de empoderamiento dentro de las comunidades. Este autor define precisamente este movimiento como un cambio desde el diseño de "cosas" (objetos) hacia el diseño de servicios que combinan elementos humanos y no humanos. Esto significa que el objeto del diseño está cambiando y no sólo se centra en productos, sino también en elementos más complejos, entrando en nuevos entornos que abarcan también la vida cotidiana y la esfera pública. De este modo, podemos encontrar muchas experiencias de diseño participativo con población vulnerable (refugiados, ancianos, enfermos, etc.) (Aldridge, 2016)

La tradición norteamericana es posterior y pone el énfasis en la participación de los usuarios, más que en el componente colaborativo o en la voluntad de empoderamiento y emancipación. Se ha desarrollado especialmente en el ámbito informático en el área de la interacción hombre-máquina, a partir del concepto de diseño centrado en el usuario que surge de la necesidad de ajustar el desarrollo tecnológico a las características y necesidades de los usuarios. Según Baek, Cagiltay, Boling y Frick (2007), en el enfoque norteamericano se ha utilizado más el diseño orientado al 
producto mientras que, en el europeo, se ha aplicado más el diseño orientado al proceso. El enfoque orientado a los procesos es más complejo ya que requiere que los diseñadores tengan en cuenta el contexto del aprendizaje humano y su comunicación. Hay que considerar no sólo los aspectos técnicos de un sistema (las herramientas, las técnicas y los procedimientos) sino también los aspectos sociales y culturales (las personas, sus roles, relaciones y tareas) y por este motivo más que hablar de diseño participativo, muchos autores usan el término de "codiseño".

Según Sanders y Stappers (2008), en el diseño centrado en el usuario, los participantes proporcionan la información para el desarrollo del sistema, la aplicación o el servicio mientras que en el diseño participativo o codiseño, los futuros usuarios participan desde el inicio en la propia concepción del producto. De este modo, la diferencia entre ambas aproximaciones aparece en las primeras fases del diseño. En el diseño centrado en el usuario, el producto a desarrollar está determinado previamente mientras que, en el codiseño, el producto final no está definido antes de iniciar la investigación y los participantes colaboran y producen conocimiento de forma conjunta. Ambos enfoques se consideran parte del diseño participativo ya que implica la aceptación de que las personas tienen percepciones diversas que afectan al uso de un producto o servicio (Sanders y Stappers, 2008). Willis y Wright (2000) distinguen entre el diseño participativo "débil" y el diseño participativo "fuerte". En el diseño participativo débil, la toma de decisiones la realizan principalmente los propios diseñadores, a pesar de que se solicita información a los usuarios utilizando diversas herramientas y técnicas mientras que, en el diseño participativo fuerte, los usuarios participan durante todo el diseño.

El diseño participativo no debe entenderse como una mera intervención de los implicados. Participar, desde esta perspectiva implica investigar, reflexionar, comprender, proponer, desarrollar y apoyar mutuamente los procesos de aprendizaje entre los participantes a lo largo de todo el proceso. La reflexión colectiva en la acción revierte sobre todos los participantes en forma de un mayor conocimiento y comprensión sobre el contexto, las prácticas que tienen lugar y los artefactos diseñados (Robertson y Simonsen, 2012). Las prácticas, de hecho, se contemplan en tanto que actividades sociales conformadas y desarrolladas en el seno de una comunidad determinada. De este modo, es la propia comunidad quien debe moldear los usos y roles de las tecnologías. Se considera que utilizando esta aproximación los productos diseñados serán más fácilmente aceptados e integrados, pero además serán más flexibles y a la vez más sólidos en el uso, accesibles para un mayor número de personas y más adaptables a situaciones cambiantes a lo largo del tiempo.

En el campo del diseño participativo confluyen nociones cercanas como la co-creación, la coproducción o el codiseño. En general, todos estos términos se refieren a acciones de creatividad colectiva y creación conjunta de conocimiento. En el ámbito social y educativo la aplicación de los métodos de codiseño tiene relación con los enfoques participativos y colaborativos de investigación, pero deriva claramente de la disciplina del diseño participativo. El elemento común a todos estos campos de aplicación es el análisis de la participación conjunta de los diferentes 
actores implicados en el uso de determinadas metodologías, productos y/o herramientas tecnológicas, la cual hace posible la trazabilidad y la interpretación de los fenómenos asociados a este uso.

\section{DISEÑO PARTICIPATIVO EN EDUCACIÓN}

En el ámbito educativo, en las últimas décadas un número creciente de autoras ha abogado por el uso de técnicas participativas en el diseño de aprendizaje (Bonsignore et al., 2013; DiSalvo et al., 2017; Druin, 2002). Algunos de los motivos que explican este giro responden al papel predominante de enfoques centrados en el estudiante, así como el reconocimiento de que nadie conoce mejor que las propias implicadas sus experiencias en torno al aprendizaje (Iversen et al., 2017).

La creciente aceptación del diseño participativo en el diseño de aprendizaje también se explica por el auge del paradigma constructivista. Hay una estrecha relación entre el diseño participativo y los enfoques constructivistas ya que en ambos se prima el proceso y la experiencia de las implicadas (Bødker et al., 1988; Gros, 2002). Desde estas perspectivas, la experiencia de aprendizaje se entiende como un proceso que se construye a lo largo del tiempo y en el que la interacción y el diálogo juegan un papel fundamental.

El diseño de aprendizaje de forma colaborativa implica generar procesos colectivos en los que las educadoras, las aprendices, así como las demás partes implicadas, tienen la posibilidad de influir y controlar el proceso de diseño y las soluciones generadas a través de este. Se trata de establecer relaciones horizontales de aprendizaje mutuo, en las que se valoran los saberes que cada parte aporta. De este modo, las partes implicadas en el proceso pueden desarrollar un sentido de propiedad y responsabilidad sobre las soluciones (Druin, 2014).

En educación formal, el diseño participativo y el codiseño se han utilizado para la definición de políticas educativas (Smith et al., 2020), currículum académico (Bovill et al., 2011; Ye et al., 2010), la ideación de actividades (Toikkanen et al., 2015), apoyar procesos de innovación (Roschelle et al., 2006), así como para diseñar materiales y herramientas de aprendizaje (Bonsignore et al., 2013). En contextos de educación fuera del aula, algunas de las iniciativas se han centrado en el diseño de instalaciones y programas educativos en instituciones culturales (Taxén, 2004), el diseño de juegos (Diaz et al., 2012; Garcés Dávila, en prensa) y en el aprendizaje de ciencias (Durall et al., 2020).

Si bien el diseño colaborativo se ha utilizado en niveles y contextos educativos diversos, un área en la que ha tenido una gran influencia es el diseño de entornos de aprendizaje mediados por tecnología. En este contexto, los enfoques participativos y de codiseño se han utilizado para involucrar a docentes y a estudiantes en la tarea de diseño de la tecnología (Cober et al., 2015, 
Bovill et al., 2011). Por otra parte, el diseño de herramientas de forma participativa se ha utilizado para apoyar competencias de aprendizaje centradas en la colaboración (Leinonen \& Durall, 2014; Spikol et al., 2009), la evaluación (Penuel et al., 2007), la reflexión (Durall et al., 2017; Leinonen et al., 2016), así como el el aprendizaje autodirigido (Laanpere et al., 2014) y abierto (Põldoja, 2016).

Diseñadoras y académicas también han justificado la implicación de diversos colectivos en el proceso de diseño de tecnología educativa reivindicando el derecho de estos a influenciar el proceso de diseño de las soluciones que impactarán sus formas de hacer y aprender. De este modo, el diseño colaborativo de entornos de aprendizaje mediados por tecnología se ha utilizado para involucrar y dar voz a grupos a los cuales se les había excluido del proceso de diseño. Algunos proyectos que se enmarcan en esta línea han buscado la participación de niñas (Iversen et al., 2017; McNally et al., 2018), personas con necesidades especiales (Frauenberger et al., 2015), así como colectivos vulnerables como migrantes (Gros \& Maniega, 2018) y jóvenes en riesgo de exclusión social (Ravenscroft et al., 2015). Las investigaciones desarrolladas a partir del análisis de estos proyectos han destacado el impacto positivo de los enfoques participativos en la confianza y la autoestima de las participantes, así como su potencial para apoyar procesos de emancipación y empoderamiento de estos colectivos.

\section{MÉTODOS DE DISEÑO PARTICIPATIVO}

Desde sus inicios, el diseño participativo se ha caracterizado por el uso de métodos que facilitan la comunicación y colaboración entre las diseñadoras y las demás partes implicadas. Así, el uso de métodos basados en juegos, el prototipado o la interpretación en los talleres participativos ha ayudado a las diseñadoras a generar un ambiente lúdico, libre de jerarquías en el que se reconocen los diferentes puntos de vista e intereses de las partes implicadas.

En los proyectos de diseño participativo es habitual encontrar una combinación de métodos cualitativos, la elección de los cuales viene dada por el contexto, las participantes, así como los objetivos, fase del proyecto y los recursos disponibles de cada proyecto. Diversos autores han propuesto clasificaciones de métodos en función de criterios como la fase temporal, el tipo de implicación entre diseñadores y participantes (ver Muller y Kuhn [1993]) o el tipo de actividad (ver Brandt et al. [2006]). En general, este tipo de técnicas buscan inspirar, así como animar a las participantes a expresarse de forma creativa y a tomar un rol activo a la hora de definir las problemáticas y generar soluciones.

El uso de métodos participativos responde a la necesidad de desarrollar una comprensión empática de las experiencias y necesidades de aquellas personas que, de forma directa o indirecta, se verán afectadas por las soluciones generadas. Según Sanders (2002), en los procesos de diseño colaborativo es importante distinguir entre lo que la gente dice, hace y siente. Los métodos basados en construir permiten acceder a información tácita, difícil de expresar de forma verbal. Al 
generar artefactos, las participantes materializan la forma en que perciben una determinada situación, así como sus preferencias y deseos en torno a esta. Mediante el análisis de los artefactos, las diseñadoras pueden entablar un proceso de diálogo con las participantes que les permita entender de forma más profunda sus necesidades y deseos.

Los escenarios de uso, los talleres futuros y los juegos de diseño son los métodos que se han utilizado ampliamente en proyectos de carácter participativo.

Los métodos de carácter narrativo como la generación de escenarios suelen utilizarse al inicio del proceso ya que permiten recabar información sobre las actividades de las participantes en su día a día (Carroll, 1999). Los talleres futuros se generaron en la década de los setenta como una técnica de participación democrática orientada a la resolución de problemas (Jungk y Müllert, 1987). Este tipo de talleres también se ha orientado a imaginar soluciones tecnológicas de forma colaborativa (Kensing y Madsen, 1991). En el diseño participativo, los juegos de diseño también se han utilizado de forma exploratoria con el fin de invitar a las participantes a reflexionar y debatir sobre futuros posibles (Brandt, 2006).

Los métodos de carácter generativo como las sondas de diseño y los prototipos también se han empleado con frecuencia en los procesos de diseño colaborativo. En esta línea, autoras como Mattelmäki y Sanders recomiendan las prácticas creativas y colaborativas (ver por ejemplo las sondas de diseño [Mattelmäki, 2006] o los enfoques centrados en crear [Sanders y Stappers, 2014]) con el fin de inspirar, fomentar el pensamiento de diseño y favorecer la reflexión con y mediante objetos por parte de las participantes.

\section{IMPLICACIONES DEL DISEÑO PARTICIPATIVO PARA LA INVESTIGACIÓN E INNOVACIÓN EN TECNOLOGÍA EDUCATIVA}

Independientemente del tipo de método que utilicemos durante el diseño, hay una serie de cuestiones que todo diseño participativo debe plantearse, nos referimos a los niveles de participación, el papel de las participantes, la capacidad de agencia de las participantes y la escalabilidad de los proyectos.

Una cuestión clave en el diseño participativo es determinar, desde el inicio, los diferentes niveles de participación y los roles que tendrán los participantes a lo largo del diseño (Bustamante et al 2018; Sabiescu et al., 2019). Existen diversas clasificaciones sobre los niveles de intervención en el diseño participativo. Bovill y Bulley (2011) establecen la "escalera de la participación" formada por ocho niveles. En el nivel más bajo- el peldaño 1- se sitúa el currículo establecido sin ningún tipo de participación de los estudiantes. En el nivel más alto- peldaño 8- se sitúa el codiseño en que los estudiantes participan junto al profesorado en el diseño del curso. Los peldaños intermedios hacen referencia a diferentes tipos de temáticas en las que pueden participar los estudiantes para diseñar 
algunos aspectos del diseño formativo. Por ejemplo, el tipo de evaluación, la elaboración de algunos contenidos, etc. En la propuesta, Bovill y Bulley (2013) no consideran que necesariamente tengamos que alcanzar el último peldaño ya que hay situaciones en que sabemos que es muy difícil. Por ejemplo, resulta complicado que las estudiantes de primer curso puedan alcanzar el último nivel en la escala de participación, pero sí que es posible si se incorporan estudiantes de cursos más avanzados que tendrán una mayor experiencia y conocimiento. En definitiva, la propuesta pretende evidenciar diferentes posibilidades y grados de implicación de las estudiantes en un proceso de codiseño.

Rinaudo y Donolo (2010), consideran que no son sólo cuestiones metodológicas las que acompañan las decisiones acerca de la composición del equipo y los grados de participación, "hay también aspectos éticos y prácticos que es necesario establecer, tales como: ¿cuáles son los compromisos que el participante debe asumir?, ¿qué tiempo le demandará?, ¿qué beneficios obtendrá con esta participación?, ¿qué tipo de información tendrá que proporcionar y de qué modo se obtendrá?, ¿qué participación tendrá en la interpretación de los datos?, ¿cuál será su participación en la elaboración del informe de investigación y cuáles serán sus derechos sobre la producción intelectual?" Rinaudo y Donolo (2010, p. 9). Si bien no existen pautas estrictas acerca del modo en que se debería responder a estas cuestiones, lo que resulta importante es plantearlas de forma explícita en las primeras etapas de la investigación.

En la investigación en diseño participativo, se ha prestado especial atención a cuestiones relacionadas con las relaciones de poder entre diseñadoras y participantes, así como la capacidad de agencia de las participantes. Desde esta perspectiva, la agencia se ha entendido como la capacidad de las personas de ejercer control y desarrollar un sentido de propiedad sobre las problemáticas y las soluciones generadas en torno a estas (Biesta y Tedder, 2006). Algunas de las estrategias utilizadas consisten en la creación de espacios "seguros" en los que las participantes se sienten valoradas y cómodas para expresar su opinión sin miedo a ser juzgadas. En este sentido, el uso de métodos creativos en los que se favorecen diversas formas de expresión es uno de los aspectos distintivos de los procesos participativos en los que se busca incluir diversas posturas, experiencias y saberes. Por otra parte, en el diseño de la tecnología de forma participativa, la capacidad de influencia y control de todas las partes implicadas durante el proceso de diseño es un aspecto crítico que conecta con la reivindicación de la democratización del proceso de diseño de tecnología (Feenberg, 2017).

Una característica de las investigaciones basadas en el diseño participativo es que se desarrollan en paralelo dos tipos de conocimientos: el codiseño (el producto) y la obtención de datos sobre el proceso participativo. Ambos están íntimamente relacionados, pero tienen diferentes objetivos. Se espera que los resultados del codiseño beneficien directamente a los participantes y a su contexto. En cambio, los resultados de la investigación sobre el codiseño tienen como objetivo construir nuevo conocimiento que permitirá la difusión y la transferencia para llevar a cabo nuevos diseños. En muchas ocasiones, estos dos niveles no se hacen explícitos ni se planifican 
previamente. Por ello, son habituales las críticas a este tipo de metodología en cuanto a la dificultad de poder replicar y escalar las investigaciones realiza das. Fallman y Stolterman (2010), argumentan que es necesario mostrar el rigor de este tipo de investigaciones en términos de coherencia interna del propio diseño. Analizar la coherencia significa examinar el modo en que se realiza un trabajo riguroso durante el proceso y, también, que el diseño exhibe cualidades que son coherentes. Por ejemplo, si realmente se basa en una epistemología que acomoda los valores democráticos, si se involucran a las partes interesadas y pueden participar en la orientación de la investigación, etc.

Se ha discutido mucho sobre la cientificidad de las investigaciones basadas en el diseño participativo (Frauenberger et al., 2015; Zimmerman et al., 2010) y existen visiones críticas sobre su uso que cuestionan la transferencia de los resultados. En esta línea, Frauenberger et al. (2015) mantienen que la investigación basada en el diseño participativo no puede ser juzgada bajo una visión positivista de la ciencia sino desde posiciones propias de epistemologías socioconstructivistas. La responsabilidad y el rigor son los aspectos clave sobre los que hay que fundamentar las aportaciones de las investigaciones basadas en el diseño participativo. Definen la responsabilidad como "la habilidad de vincular de forma transparente el trabajo colaborativo realizado durante la investigación participativa con las decisiones y los resultados obtenidos" Frauenberger et al (2015, p. 94).

Tal y como hemos señalado a lo largo del artículo, uno de los aspectos más interesantes del diseño participativo es la reflexión en la acción que revierte en todas las participantes en forma de un mayor conocimiento y comprensión sobre el contexto, las prácticas que tienen lugar y los artefactos diseñados. En definitiva, tiene mucho interés para mejorar la práctica y la investigación sobre los procesos de cambio educativo. Sin embargo, es necesario seguir avanzando para dar respuesta a los muchos desafíos importantes que hemos ido señalando: la configuración de los equipos y agentes implicados, la selección de las técnicas e instrumentos a utilizar en las diferentes fases, el tipo de artefactos generados y la sostenibilidad de los productos cuando finalizan los proyectos.

\section{REFERENCIAS}

Aldridge, J. (2016). Participatory research: Working with vulnerable groups in research and practice. Bristol: Policy Press.

Baek, E. O., Cagiltay, K., Boling, E. y Frick, T. (2007). User-centered design and development. Handbook of research on educational communications and technology. En J.M. Spector, D. Merrill MD, J. van Merrienboer, M. Driscoll. Handbook of research on educational communications and technology (pp. 660-668). Mahwah: Lawrence Erlbaum Associates. https://doi.org/10.4324/9780203880869.ch49 
Biesta, G. y Tedder, M. (2006). How Is Agency Possible? Towards an Ecological Understanding of Agency-as-Achievement. Learning Lives: Learning, Identity, and Agency in the Life Course. Working Paper Five, Exeter: Teaching and Learning Research Programme.

Bonsignore, E., Ahn, J., Clegg, T., Guha, M. L., Hourcade, J. P., Yip, J. C. y Druin, A. (2013). Embedding Participatory Design into Designs for Learning: An Untapped Interdisciplinary Resource?. En N. Rummel, M. Kapur, M. Nathan y S. Puntambekar (Eds.), To See the World and a Grain of Sand: Learning across Levels of Space, Time, and Scale: CSCL 2013 Conference Proceedings, Volume 1 (pp.549-556).

Bovill, C. y Bulley, C.J. (2011) A model of active student participation in curriculum design: exploring desirability and possibility. En C. Rust (ed.) Improving Student Learning: Global Theories and Local Practices: Institutional, Disciplinary and Cultural Variations. (pp. 176-188). Oxford: Oxford Brookes University.

Bovill, C., Cook-Sather, A. y Felten, P. (2011). Students as co-creators of teaching approaches, course design, and curricula: implications for academic developers. International Journal for Academic Development, 16(2), 133-145. https://doi.org/10.1080/1360144X.2011.568690

Bødker, S., Ehn, P., Knudsen, J., Kyng, M. y Madsen, K. (1988). Computer support for cooperative design. En Proceedings of the 1988 ACM conference on Computer-supported cooperative work (pp. 377-394). https://doi.org/10.1145/179606.376379

Brandt, E. (2006). Designing exploratory design games: a framework for participation in Participatory Design?, En Proceedings of the ninth conference on Participatory design: Expanding boundaries in design, 1 (pp. 57-66). https://doi.org/10.1145/1147261.1147271

Bustamante, A. M., Degbelo, A. y Kray, C. (2018). Exploring Forced Migrants (Re) settlement \& the Role of Digital Services. In Proceedings of 16th European Conference on ComputerSupported Cooperative Work-Exploratory Papers. European Society for Socially Embedded Technologies (EUSSET). https://doi.org/10.18420/ecscw2018

Carroll, J. M. (1999). Five Reasons for Scenario-Based Design. Proceedings of the 32nd Hawaii International conference on system Sciences. https://doi.org/10.1109/HICSS.1999.772890

Cober, R., Tan, E., Slotta, J., So, H. J. y Könings, K. D. (2015). Teachers as participatory designers: Two case studies with technology-enhanced learning environments. Instructional Science, 43(2), 203-228. https://doi.org 10.1007/s11251-014-9339-0

Diaz, P., Paredes, P., Alvarado, D. y Giaccardi, E. (2012). Co-designing social games with children to support non formal learning. En 2012 IEEE 12th International Conference on Advanced Learning Technologies (pp. 682-683). IEEE. https://doi.org/ 10.1109/ICALT.2012.22 
DiSalvo, B., Yip, J., Bonsignore, E. y DiSalvo, C. (Eds.). (2017). Participatory design for learning: Perspectives from practice and research. New York: Routledge. https://doi.org/10.4324/9781315630830

Druin, A. (2002). The role of children in the design of new technology. Behaviour and information technology, 21(1), 1-25. https://doi.org/10.1080/01449290110108659

Druin, A. (2014). Inclusive ownership of participatory learning. Instructional Science, 42(1), 123126. https://doi.org 10.1007/s11251-014-9339-0

Durall, E., Bauters, M., Hietala, I., Leinonen, T. y Kapros, E. (2020). Co-creation and co-design in technology-enhanced learning: Innovating science learning outside the classroom. Interaction Design and Architecture (s), 42, 202-226. https://bit.ly/2J11w5H

Durall, E., Leinonen, T., Gros, B. y Rodriguez-Kaarto, T. (2017). Reflection in learning through a selfmonitoring device: Design research on EEG self-monitoring during a study session. Designs for Learning, 9(1), 10-20. https://doi.org 10.16993/dfl.75

Ehn, P. (1988). Work-Oriented Design of Computer Artefacts. Hillsdale, NJ: Lawrence Erlbaum Associates.

Ehn, P. (2008). Participation in design things. In Proceedings of the 10th Anniversary Conference on Participatory Design. New York: ACM.

Fallman, D. y Stolterman, E. (2010). Establishing criteria of rigour and relevance in interaction design research. Digital Creativity, 21(4), 265-272. https://doi.org 10.1080/14626268.2010.548869

Feenberg, A. (2017). A Critical Theory of Technology. En U. Felt, R. Fouché, \& L. Smith-Doerr (Eds.), The Handbook of Science and Technology Studies (pp. 635-663). Cambridge, MA: MIT press. https://doi.org 10.4135/9781446282229.n9

Frauenberger, C., Good, J., Fitzpatrick, G. y Iversen, O. S. (2015). In pursuit of rigour and accountability in participatory design. International Journal of Human-Computer Studies, 74, 93-106. https://doi.org 10 1016/j.ijhcs.2014.09.004

Garcés Dávila, A.L. (en prensa). Revitalización de saberes ancestrales y diseño participativo. Codiseño de un juego comunitario. PDC 2020.

Gros, B. (2002). Constructivismo y diseño de entornos virtuales de aprendizaje. Revista de Educación, mayo-agosto, 225. https://bit.ly/2V1sb4r

Gros, B. y Maniega, D. (2018). Co-Designing Apps to Support the Learning Needs of Migrants in Barcelona (Spain). Journal of Interactive Learning Research, 29(3), 465-480. https://bit.ly/2Jae5LY 
Frauenberger, C., Good, J. y Alcorn, A. (2012, June). Challenges, opportunities and future perspectives in including children with disabilities in the design of interactive technology. En Proceedings of the 11th International Conference on Interaction Design and Children (pp. 367-370).

Iversen, O. S., Smith, R. C. y Dindler, C. (2017). Child as protagonist: Expanding the role of children in participatory design. En Proceedings of the 2017 Conference on Interaction Design and Children (pp. 27-37). http://dx.doi.org/10.1145/3078072.3079725

Jungk, R. y Müllert, N. (1987). Future workshops: How to Create Desirable Futures. London: Institute for Social Invention.

Kensing, F. y Madsen, K. H. (1991). Generating visions: Future workshops and metaphorical design. En J. Greenbaum y M. Kyng (Eds.), Design at work: Cooperative Design of Computer Systems (pp.155-168). Hillsdale NJ: Lawrence Erlbaum.

Laanpere, M., Pata, K., Normak, P. y Põldoja, H. (2014). Pedagogy-driven design of digital learning ecosystems. Computer Science and Information Systems, 11(1), 419-442. https://doi.org/10.2298/CSIS121204015L

Leinonen, T., y Durall, E. (2014). Design thinking and collaborative learning. Comunicar. Media Education Research Journal, 22(1). https://doi.org/10.3916/C42-2014-10.

Leinonen, T., Keune, A., Veermans, M. y Toikkanen, T. (2016). Mobile apps for reflection in learning: A design research in K-12 education. British Journal of Educational Technology, 47(1), 184202. https://doi.org/10.1111/bjet.12224

Mattelmäki, T. (2006). Design probes. Ph.D. Aalto University School of Art and Design.

McNally, B., Kumar, P., Hordatt, C., Mauriello, M. L., Naik, S., Norooz, L., Shorter, A., Colub, E. y Druin, A. (2018). Co-designing mobile online safety applications with children. En Proceedings of the 2018 CHI Conference on Human Factors in Computing Systems (pp.1-9). https://doi.org/ 10.1145/3173574.3174097

Muller, M. J. y Kuhn, S. (1993). Participatory design. Communications of the ACM, 36(6), 24-28.

Penuel, W. R., Roschelle, J. y Shechtman, N. (2007). Designing formative assessment software with teachers: An analysis of the co-design process. Research and practice in technology enhanced learning, 2(01), 51-74. https://doi.org/ 10.1142/S1793206807000300

Põldoja, H. (2016). The Structure and Components for the Open Education Ecosystem-Constructive Design Research of Online Learning Tools. Ph.D. Aalto University School of Art and Design. Helsinki: Aalto University Publication Series. https://aaltodoc.aalto.fi/handle/123456789/23535 
Rinaudo, M. y Donolo, D. (2010). Estudios de diseño. Una perspectiva prometedora en la investigación educativa RED. Revista de Educación a Distancia, 22, 11-29. https://bit.ly/33iGF4C

Robertson, T. y Simonsen, J. (2012). Challenges and opportunities in contemporary participatory design. Design Issues, 28(3), 3-9. https://doi.org/10.1162/DESI_a_0015

Roschelle, J., Penuel, W. y Shechtman, N. (2006). Co-design of innovations with teachers: Definition and dynamics. En Proceedings of the 7th international conference on Learning sciences (pp. 606-612). International Society of the Learning Sciences.

Sabiescu, A., de Moor, A. y Memarovic, N. (2019). Opening up the culture black box in community technology design. Al \& Soc 34, 393-402 (2019). https://doi.org/10.1007/s00146-01900904-z

Sanders, E. (2002). From user-centered to participatory design approaches. En Design and the social sciences (pp.18-25). CRC Press.

Sanders, E. y Stappers, P. J. (2008). Co-creation and the new landscapes of design. CoDesign, 4(1), 5-18. https://doi.org/10.1080/15710880701875068

Sanders, E. B. N., Brandt, E. y Binder, T. (2010). A framework for organizing the tools and techniques of participatory design. En Proceedings of the 11th biennial participatory design conference (pp. 195-198).

Sanders, E. B. N. y Stappers, P. J. (2014). Probes, toolkits and prototypes: three approaches to making in codesigning. CoDesign, 10(1), 5-14. https://doi.org/ 10.1080/15710882.2014.888183.

Smith, R. C., Bossen, C., Dindler, C. y Iversen, O. S. (2020). When Participatory Design Becomes Policy: Technology Comprehension in Danish Education. En Participatory Design Conference Biennial Participatory Design Conference. ACM. https://doi.org/ $10.1145 / 3385010.3385011$

Spikol, D., Milrad, M., Maldonado, H. y Pea, R. (2009). Integrating co-design practices into the development of mobile science collaboratories. En 2009 Ninth IEEE International Conference on Advanced Learning Technologies (pp.393-397). IEEE. https://doi.org/ 10.1109/ICALT.2009.175

Taxén, G. (2004). Introducing participatory design in museums. En Proceedings of the eighth conference on Participatory design: Artful integration: interweaving media, materials and practices, 1 (pp. 204-213). https://doi.org/10.1145/1011870.1011894 
Toikkanen, T., Keune, A. y Leinonen, T. (2015). Designing Edukata, a participatory design model for creating learning activities. En Re-engineering the Uptake of ICT in Schools (pp.41-58). Springer. https://doi.org/ 10.1007/978-3-319-19366-3_3

Ravenscroft, A., Rainey, C., Dellow, J., Brites, M. J., Auwärter, A., Balica, M., Rees A., Camilleri A. F., Jorge, A., Dahn, I. y Fenech J. (2015). RadioActive Europe: promoting engagement, informal learning and employability of at risk and excluded people across Europe through internet radio and social media. https://doi.org/10.13140/RG.2.1.4645.4888

Willis, J. y Wright, K. E. (2000). A general set of procedures for constructivist instructional design: The new R2D2 model. Educational Technology, 40(2), 5-20. https://bit.ly/33iGXse

Ye, X., Zhang, B. y Chia, P. (2010). A tripartite model of co-designing for an iMVT integrated science curriculum. En S. L. Wong, S.C. Kong y F.-Y. Yu (Eds.), Proceedings of the 18th International Conference on Computers in Education (pp. 145-152). Malaysia: Asia-Pacific Society for Computers in Education.

Zimmerman, J., Stolterman, E. y Forlizzi, J. (2010). An analysis and critique of Research through Design: towards a formalization of a research approach. In proceedings of the 8th ACM conference on designing interactive systems (pp. 310-319). ACM. https://doi.org/ 10.1145/1858171.1858228

\section{Para citar este artículo:}

Gros, B., y Durall, E. (2020). Retos y oportunidades del diseño participativo en tecnología educativa. Edutec. Revista Electrónica De Tecnología Educativa, (74), 12-24.

https://doi.org/10.21556/edutec.2020.74.1761 